\title{
Utilization of Artificial Intelligence in Medical Image Analysis for COVID-19 Patients Detection
}

\author{
Mohammed Baz $^{1, *}$, Hatem Zaini ${ }^{1}$, Hala S. El-sayed ${ }^{2}$, Matokah AbuAlNaja ${ }^{3}$, Heba M. El-Hoseny ${ }^{4}$ and \\ Osama S. Faragallah

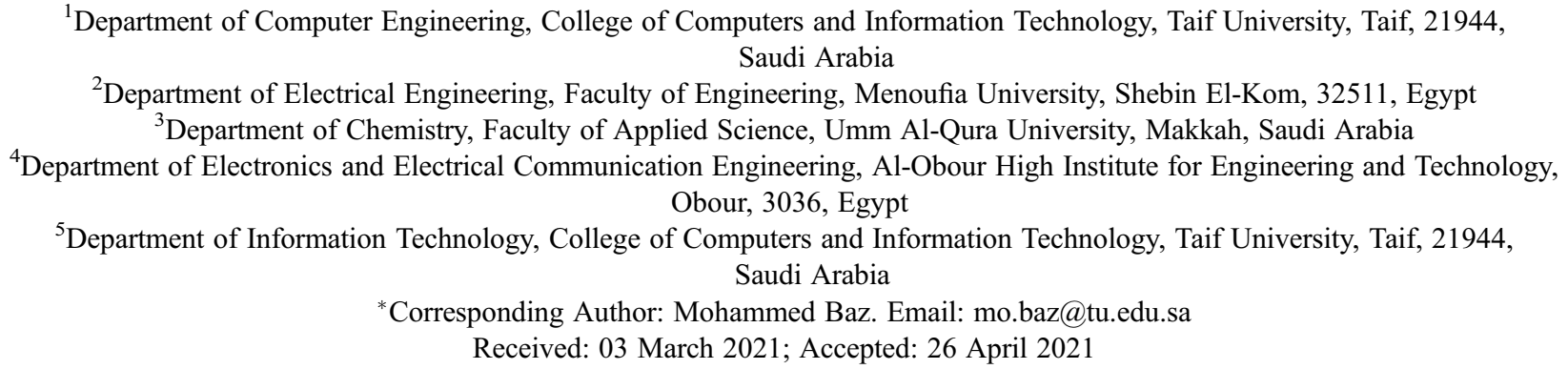

\begin{abstract}
In the era of medical technology, automatic scan detection can be considered a charming tool in medical diagnosis, especially with rapidly spreading diseases. In light of the prevalence of the current Coronavirus disease (COVID-19), which is characterized as highly contagious and very complicated, it is urgent and necessary to find a quick way that can be practically implemented for diagnosing COVID-19. The danger of the virus lies in the fact that patients can spread the disease without showing any symptoms. Moreover, several vaccines have been produced and vaccinated in large numbers but, the outbreak does not stop. Therefore, it is urgent and necessary to find a quick way that can be practically implemented for diagnosing COVID-19 cases. One of the most important ways to combat this disease is the early detection of the virus by using technology to identify and isolate patients. The combined results of recent researches showed that both CT and CXR scan correctly diagnosed COVID-19 in $87 \%$ and $80 \%$ of infected people. From these perspectives, this research paper aims to employ a deep learning model using the convolutional neural network $(\mathrm{CNN})$ to detect and diagnose COVID-19 from both CT and CXR scans. The CNN is being used for features extraction and then detect COVID-19 cases in a little bit of time. Dataset collections of CT and CXR scans are being applied for examining the proposed CNN-based COVID-19 detection model. The results show that the proposed CNN-based COVID-19 detection model can achieve an accuracy of $99 \%$, effectively speeding up the diagnosis and treatment of COVID-19 patients from CT and CXR scans.
\end{abstract}

Keywords: CT; CXR; CNN; COVID-19

This work is licensed under a Creative Commons Attribution 4.0 International License, which permits unrestricted use, distribution, and reproduction in any medium, provided the original work is properly cited. 


\section{Introduction}

At the end of 2019, a new wave of respiratory infections from the Coronavirus family has spread worldwide, dubbed COVID-19. It is transmitted from a person to another person at an unprecedented speed, and the World Health Organization (WHO) has confirmed that it is a dangerous infection $[1,2]$. The medical reports have stated that COVID-19 is responsible for severe digestive and respiratory diseases in humans and mammals [3-5]. Moreover, it has devastated the economy and health care systems in developing countries because of overcrowding in hospitals that contain a limited number of units and intensive care rooms. Up to date, the statistics of COVID-19 around the world counts confirmed cases close to $110 \mathrm{M}$ compared to $60.5 \mathrm{M}$ recoveries and $2.38 \mathrm{M}$ deaths.

All studies indicated the need for early diagnosis to control the spread of COVID-19 and increase the survival chances [6,7]. The WHO has recommended a standard detection method using nucleic acid of the virus in real-time, called polymerase chain reaction (RT-PCR) [8-10]. The RT-PCR method has proven a high degree of quality in detecting infected persons. However, many countries in which the infection has spread widely cannot provide sufficient RT-PCR tests for the entire population due to the high cost of the test. Therefore, several restrictions have been placed on the pathological examination laboratories, and it has been recommended that tests be performed only on suspects' cases with apparent symptoms. Unfortunately, the RT-PCR result takes at least 8 hours, making it inconvenient, especially in emergencies and large numbers of infected persons.

A computer-aided diagnosis (CAD) scheme based on medical image processing has emerged as an alternative, fast, and more reliable method for detecting COVID-19. It has been found that medical imaging techniques have higher sensitivity compared to RT-PCR like chest computed tomography (CT), X-ray (CXR), magnetic resonance imaging (MRI), ultrasound, and positron emission tomography (PET) [11-13].

Although radiologists can analyze medical images through visually scanning, it is difficult for them to realize the minute details, which could raise diagnoses errors, especially in the early stages of some cases. Typically, all hospitals worldwide usually perform CT and CXR scans to examine lung abnormalities [14-16]. CXR scan is utilized to check dense tissues, while the CT scan is employed to catch soft tissues, bones, and blood vessels. The most widely scan utilized in medical centers is CXR due to its simplicity, ease of operation, low cost, low radiation to which the patient is exposed, and much smaller and less complex equipment than others. On the other hand, CT is becoming a popular tool in clinical diagnosis to obtain 3D visualization of internal tissues and extra cost and radiation.

With the continuous increase in COVID-19 cases, several methods have been suggested, either by using the CXR in suspected cases and the CT to follow up patients to ensure the lungs integrity during the infection period [17-20]. Nonetheless, the probability of COVID-19 cases misclassifications increases the number of CT scans that need to be evaluated rapidly. Moreover, it is difficult to distinguish the pneumonia of a person with COVID-19 from pneumonia caused by other infection, which wastes a lot of time and efforts in diagnosis.

To overcome the fore-mentioned challenge, modern analysis in professional healthcare systems has started using intelligent approaches to detect COVID-19 cases based on deep learning schemes. Artificial intelligence (AI) technology can access and process large amounts of data which has attracted the interest of many researchers. Intelligent approaches analyze disease characteristics automatically and provide radiologists with valuable decisions for a more accurate diagnosis of COVID-19. It can classify CT chest images in record time, monitor the epidemic, and evaluate clinical trials by analyzing epidemiological data [21-25].

The strategy for this work is to design and investigate a CNN model to detect COVID-19 patients accurately. The CNN model consists of two neural networks, one for image feature extraction and the other for image classification. The designed model supports an early diagnosis of COVID-19 cases for thousands of medical images in a very short time with high resolution and fewer network layers than the pre-trained learning models. Moreover, the model has been trained on CXR and CT dataset arrangements 
to classify the normal cases from COVID-19 infection and pneumonia cases to fight the spread of the epidemic. The performance of the proposed model is evaluated in terms of specificity, sensitivity, precision, the Matthews correlation coefficient (MCC), and negative predictive value (NPV).

The paper rest is arranged as follows. Section 2 introduces the related works. Section 3 explores the main work methodology. The suggested algorithm is explained in Section 4. Section 5 explores the evaluation matrices analysis and their numerical results. The research is concluded in Section 6.

\section{Related Works}

Numerous studies have been conducted on AI techniques for various fields and purposes. During the last decades, many scientists have been interested in integrating the medical field with AI techniques for images rapid interpretation. With the COVID-19 epidemic, it is required to find an accurate and effective diagnostic strategy to detect COVID-19 cases.

In Wang et al. [26], COVID-Net is designed to detect four classes of the dataset: COVID-19 infection, Pneumonia Viral, Pneumonia bacterial, and normal. The overall accuracy is close to $83.5 \%$ for the four classes and 92.4\% in classifying COVID-19. Another system based on a deep convolutional network for COVID-19 detection using the CXR is presented in Kumar et al. [27]. The system is enrolled and tested using a dataset collected from Kaggle, GitHub, and Open-I repository. The COVID-19 detection accuracy is close to $95.38 \%$. However, in Khan et al. [28], some of the deep-learning models were investigated for the diagnosis process of COVID-19 using the CXR and reported that VGG-16 and VGG-19 schemes have superior performance. The addition rule was conducted as a novel CNN architecture with a performance close to $98.96 \%$ [29].

Nour et al. [30] built a CNN model trained from scratch with a five cascading convolutional layered. The discriminative features were extracted using the proposed model to supply machine learning schemes like k-NN, SVM, and DT. The SVM classifier produced an accuracy of 98.97\%. A Dark CovidNet model based on deep learning was proposed for COVID-19 detection using the CXR in Ozturk et al. [31]. The classification results are investigated through 3 dataset classes which indicated $87.02 \%$ accuracy. A CoroNet model for COVID-19 detection based on Xception architecture and pre-trained using CT and CXR datasets is presented in Khan et al. [32]. The architecture results are obtained for four classes and three classes with $89.6 \%$ and $95 \%$ accuracy. Two datasets are trained using different pre-trained deep learning models in Apostolopoulos et al. [33]. The data set collection contains normal, bacterial pneumonia and COVID-19 cases. The model accuracy is close to $98.75 \%$.

$\mathrm{Xu}$ et al. [34] designed a screening scheme to differentiate COVID-19 early from Influenza-A (viral pneumonia). The model employed 618 pulmonary CT samples and gave an accuracy of $86.7 \%$. Researchers trained a ResNet50 model (COVNet) to identify COVID-19 cases from pneumonia and nonpneumonia cases using 4356 chest CT images in Li et al. [35]. The COVNet model results gave a sensitivity of $90 \%$. A Deep Pneumonia system based on deep learning to detect COVID-19 cases is proposed in Ying et al. [36]. The system is examined with Chest CT images which demonstrated an accuracy of $86.0 \%$. A Bayesian classifier based on deep learning to estimate model uncertainty using the transfer learning method for COVID-19 X-Ray images is introduced in Ghoshal et al. [37]. The Bayesian inference increases the detection accuracy with the standard VGG16 scheme from $85.7 \%$ to $92.9 \%$. A CVDNet model to classify COVID-19 infection using the CXR is introduced in Ouchichaa et al. [38]. It uses two parallel levels with distinct kernel sizes to catch the local and global inputs based on the residual neural network. The experimental results achieved a promising classification performance. Several new approaches are developed and investigated using the CNN model to detect and classify the CXR of COVID-19 cases in Heidari et al. [39]. The system reported higher classification performance in COVID-19 detection. A light CNN scheme based on SqueezeNet for COVID-19 discrimination using CT 
images is designed in Polsinellia et al. [40]. The results have an accuracy of $85.03 \%$. The model can analyze huge images every day with limited hardware resources.

\section{Methodology}

This section is devoted to providing detailed information about the work architectures materials and dataset description. Then, a brief introduction to CNN deep learning for COVID-19 classification is presented. Finally, the general information about the CNN model is given.

\subsection{Materials Description}

The proposed model is evaluated using the COVID-19 radiography database obtained from the Kaggle datasets [41,42], updated continuously to reflect the different medical cases related to the disease. The applied dataset total number is 1208 scans, as explored in Tab. 1. The dataset composed of CXR and CT scans for confirmed COVID-19, negative COVID-19, and pneumonia patients and used for the training and testing phases. The datasets are divided randomly into two groups; the first group is $80 \%$ of materials and used for training the CNN model, which is repeated ten times. The second group is the remaining $20 \%$ and used for the testing purpose. Figs. 1 and 2 illustrate samples of the studied cases that shows CXR and CT images of COVID-19, non-COVID-19, and pneumonia cases.

Table 1: Dataset description

\begin{tabular}{ll}
\hline Total number of Images & 1208 \\
\hline COVID-19 & 400 \\
Pneumonia & 402 \\
non-COVID & 406 \\
Training Dataset & $966(80 \%)$ \\
Testing dataset & $242(20 \%)$ \\
\hline
\end{tabular}

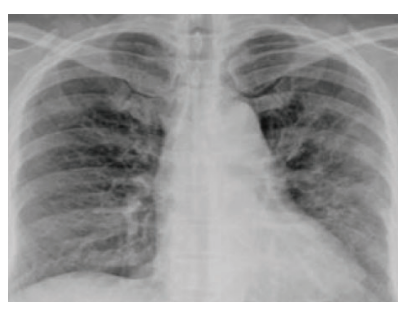

(a) COVID-19

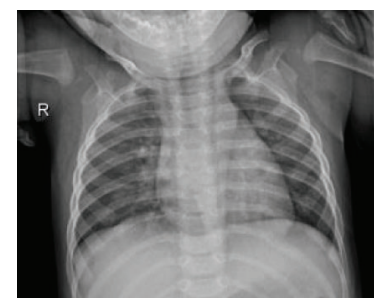

(b) non COVID-19

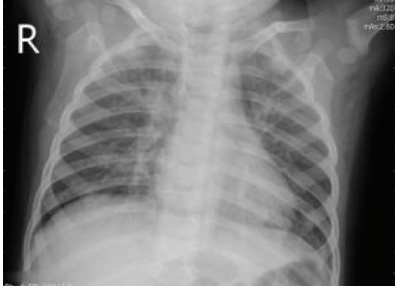

(c) pneumonia

Figure 1: Samples of X-ray images (a) COVID-19, (b) non COVID-19, (c) pneumonia

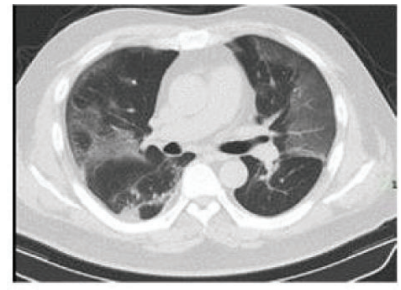

(a) COVID-19

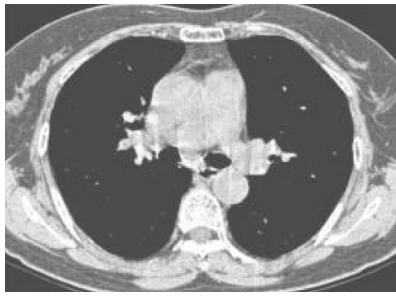

(b) non COVID-19

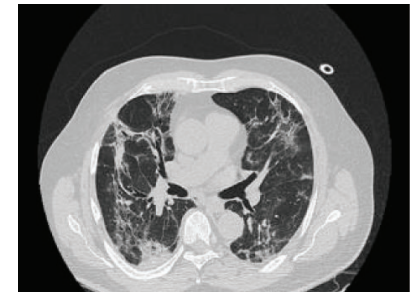

(c) pneumonia

Figure 2: Samples of CT images (a) COVID-19, (b) non COVID-19, (c) pneumonia 


\subsection{CNN Architecture}

Deep learning is a subfield of AI neural network with multilayers and can be defined as an algorithm that performs machine learning calculations in many layers like the brain function. High accuracy of deep learning can be obtained with increasing layer numbers. Recently, deep learning techniques have been applied in several areas such as social media, e-commerce, E-mail filtering, search engine matching, smartphones, face recognition, detection, object recognition, and classification.

The CNN is a deep neural network with at least one convolution layer or a particular deep learning type with multilayer, which achieved a great performance in medical analysis. The main layers are convolution, rectified linear unit (ReLU), Pooling, normalization, and fully connected (FC) layers, where the classification process occurs, as shown in Fig. 3. The CNN is implemented in two phases: extract features from images in the training process and the other to classify images [43]. The CNN main concept is to extract the local features in a high layer and then move the extracted features to lower layers for obtaining more complex features. The convolutional layer includes a set of kernels that convolve an entire input using "stride(s)" for determining feature maps which can decrease input volume dimensions. The convolutional layer is operated as $[44,45]$ :

$F(i, j)=(I * K)(i, j)=\sum \sum I(i+m, j+n) K(m, n)$

where $(I * K)$ represents the convolutional layer operation, $I$ is the input matrix, $K$ is the 2D filter of $m \times n$ size, and $F$ is a 2D feature output map. ReLU layer is used to increase the feature maps nonlinearity and calculate the activation with an input threshold at zero, which can be expressed as:

$F(X)=\max (0, X)$

The pooling layer downsampled the input object dimensions to reduce the number of image parameters but retains the important information and has different types: max-Pooling, average-Pooling, and sumPooling. The most common type is the max-pooling which picks the largest element in the rectified feature map. Finally, the FC layer is applied to make a final decision using the soft-max or sigmoid activation function to classify the output features.

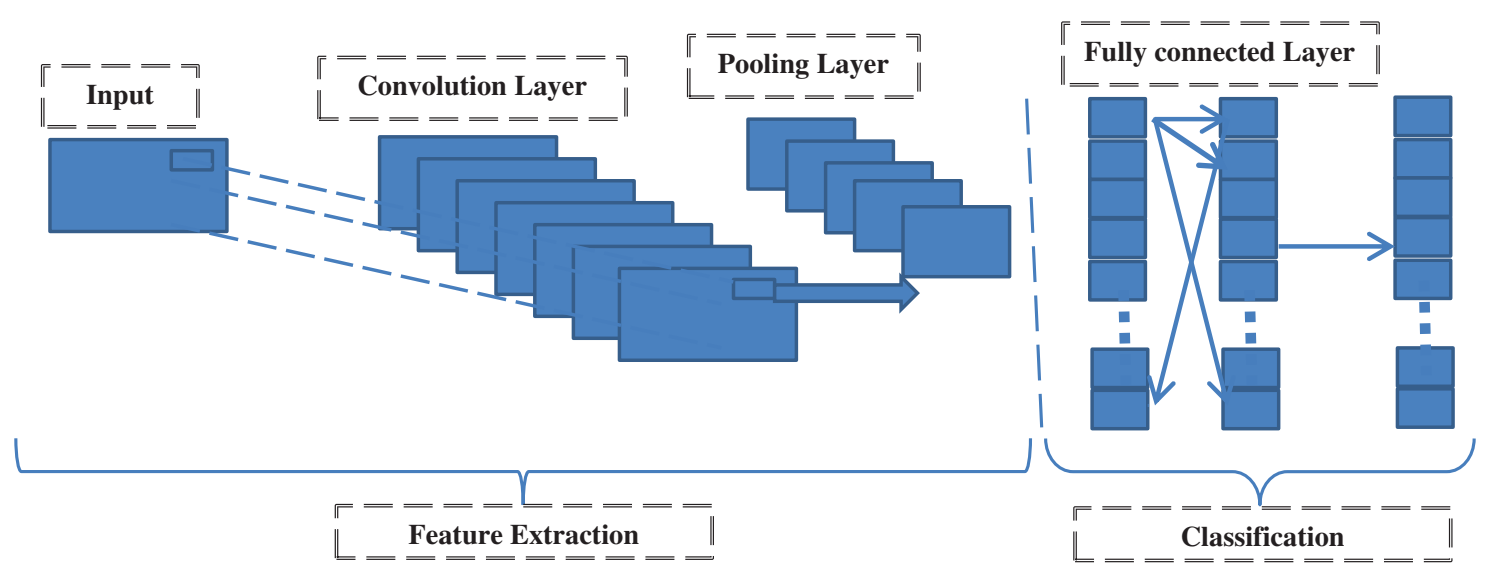

Figure 3: A schematic diagram of a typical CNN architecture

\section{The Proposed Algorithm}

Due to the complications of medical image analysis and the diagnosing difficulty of COVID-19, the degree of infection increased. The proposed CNN-based COVID-19 detection model is shown in 
Figs. 4, and 5 shows its block diagram. The network consists of 11 layers: five convolutional layers, five pooling layers, and one FC output layer with the soft-max function. The convolutional stage consists of three layers; convolutional layer, batch normalization layer, and RELU activation function with filters size of 16, 32 and 64, and 128. The convolutional layer of a size of $3 \times 3$ kernels is applied for extracting features that are carried out using the ReLU function. Then, the pooling layer decreases the image size and merges the neighbouring pixels of a specific region of the input image into a single representative value. The max-pooling layer with a size of $2 \times 2$ kernels is applied. Tab. 2 shows a detailed summary of the proposed CNN network. Finally, the classification layer consists of an FC layer and soft-max activation function to transform fully connected to several classes by predicting the target category according to the three categories (COVID-19, non-COVID-19, and pneumonia).

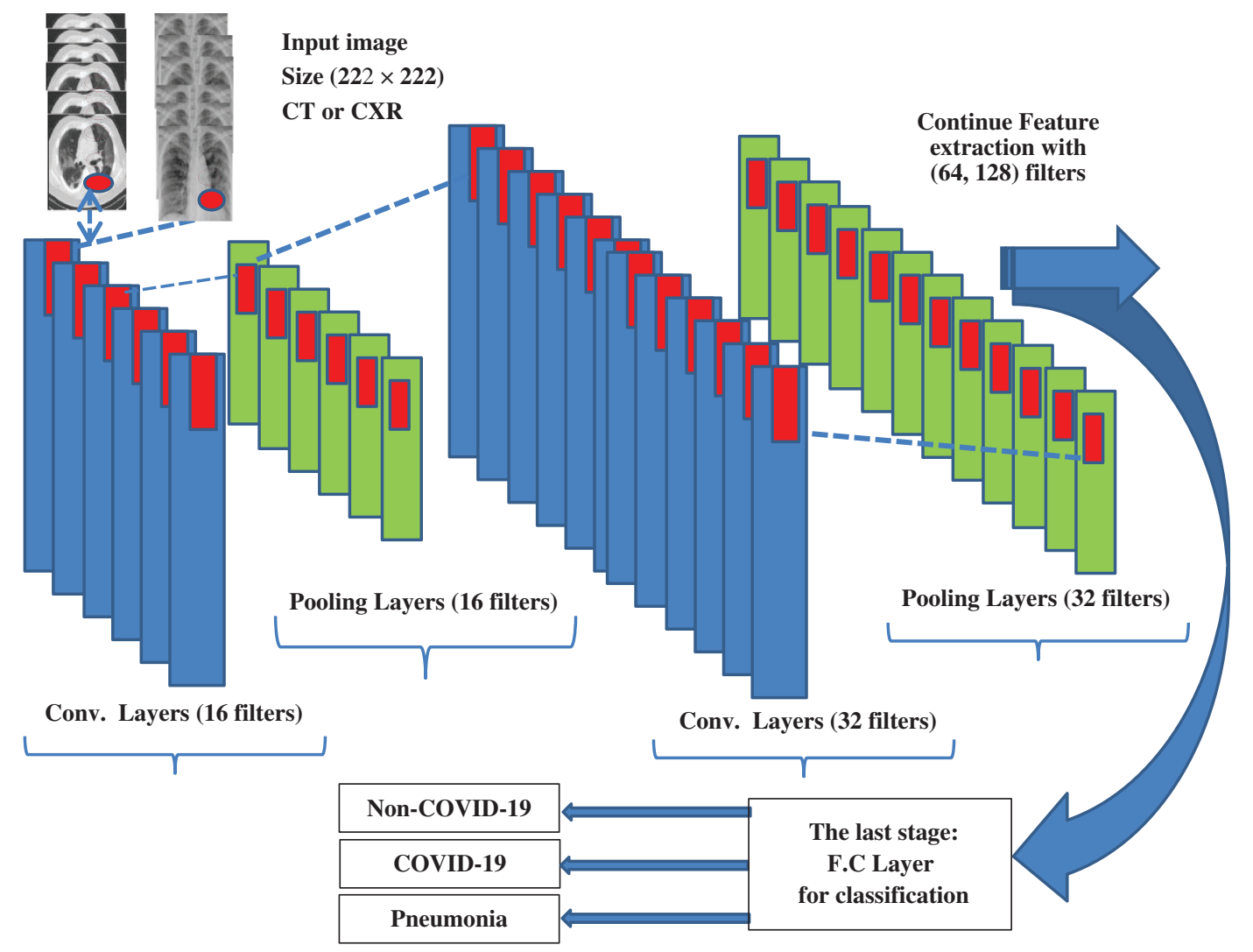

Figure 4: The architecture of the proposed CNN-based COVID-19 detection model

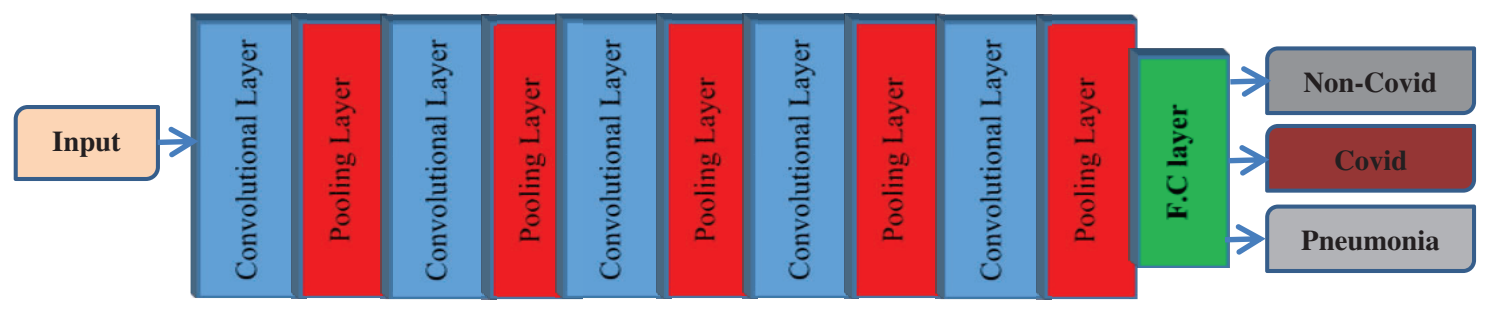

Figure 5: The block diagram of the proposed CNN-based COVID-19 detection model 
Table 2: A detailed summary of the proposed CNN-based COVID-19 detection model

\begin{tabular}{lll}
\hline Layer Name & Kernel Size & Output Size \\
\hline Conv1 layer & $3 * 3$ & $(222,222,8)$ \\
Pooling 1 layer & $2 * 2$ & $(111,111,8)$ \\
Conv 2 layer & $3 * 3$ & $(109,109,16)$ \\
Pooling 2 layer & $2 * 2$ & $(54,54,16)$ \\
Conv 3 layer & $3 * 3$ & $(52,52,32)$ \\
Pooling 3 layer & $2 * 2$ & $(26,26,32)$ \\
Conv 4 layer & $3 * 3$ & $(24,24,64)$ \\
Pooling 4 layer & $2 * 2$ & $(12,12,64)$ \\
Conv 5 layer & $3 * 3$ & $(10,10,128)$ \\
Pooling 5 layer & $2 * 2$ & $(5,5,128)$ \\
Fully Connected & & $(128)$ \\
(Classification layer) & & $(2)$ or $(3)$ \\
\hline
\end{tabular}

\section{Simulation Results}

The proposed model is suggested to rapidly detect COVID-19 cases by combining the pre-trained CNN and a binary classifier. In the proposed work, the dataset is divided into $80 \%$ for training and $20 \%$ for testing with pre-trained CNN model using VGG-16, Google-Net, and ResNet-50 models. Then, deep features were fused and ranked. In the fusion process, the work took into consideration the correlation values between features. The proposed model is evaluated by comparing the outcomes with the ground truth dataset and carrying out statistical measurements such as accuracy, sensitivity, specificity, F1-score, precision, Negative Predictive Value (NPV), and Matthews Correlation Coefficient (MCC). The utilized metrics for $\mathrm{N}$ samples are:

True Positive (TP): It counts the cases classified as patients by both the proposed model and clinicians.

True Negative (TN): It counts the cases classified as healthy by both the proposed model and clinicians.

Conversely, False Positive (FP) and False Negative (FN): count the number of cases that our model classifies as patients (healthy) whereas clinicians diagnosed them as (healthy or pneumonia) infected.

Accuracy: It is the ratio of the total cases (agreed cases from both models' expectations and clinicians' diagnoses) to the total cases seen by the proposed model or verifies how many cases are correctly classified and can be mathematically expressed as:

Accuracy $=\frac{T P+T N}{T P+F P+T N+F N}$

Specificity: The ratio between the classified cases by the model and clinicians as true to the sum of the positive cases predicted by the model or the correct rate of recognition of negative samples.

Specificity $=\frac{T N}{F P+T N}$ 
Sensitivity: It is the rate of recognition for positive samples successfully.

Sensitivity $=\frac{T P}{T P+F N}$

Precision: It estimates the model performance's precise degree by examining the correct, true positives from the predicted ones.

Precision $=\frac{T P}{T P+F P}$

NPV: It represented a negative test result and calculated the probability that a person is not affected.

$N P V=\frac{T N}{T N+F N}$

F1-Score: It tries to find a balance between sensitivity and precision, or it is the function of sensitivity and precision.

$\mathrm{F} 1-$ score $=2 \times \frac{\text { Pre. } \times \text { Sen. }}{\text { Pre. }+ \text { Sen. } .}=\frac{2 \mathrm{TP}}{(2 \mathrm{TP}+\mathrm{FP}+\mathrm{FN})}$

MCC: It measures the quality of binary classifications in machine learning and ranges from -1 to 1 .

$M C C=\frac{(T P \times T N-F P \times F N)}{\sqrt{(T P+F P)} \times(T P+F N) \times(T N+F P) \times(T N+F N)}$

Through analyzing the obtained results values, the estimated statistical metrics will be a useful aid for technicians to prove if the detected case is classified as an infected case or misclassified as a normal case. The classification performance of the proposed CNN-based COVID-19 detection model in terms of various measures like accuracy, specificity, sensitivity, and precision, NPV, F1-score, and MCC using CT scans is summarized in Tab. 3. The model outcomes demonstrated superior performance in the classification of COVID-19, non-COVID-19, and pneumonia using CT scans. The model has 100\% overall accuracy, $100 \%$ specificity, $100 \%$ sensitivity, $100 \%$ precision, $100 \%$ NPV, 1 for F1-score, and 1 for MCC. However, the classification performance in COVID-19 and non COVID-19 detection using CT scans demonstrated $98.85 \%$ overall accuracy, $100 \%$ specificity, $99.33 \%$ sensitivity, $99 \%$ precision, $99.32 \%$ NPV, $99.62 \%$ for F1-score and $99.33 \%$ for MCC. Therefore, the proposed network gives high results with several CT input scans.

Table 3: The proposed CNN-based COVID-19 detection model in classification using CT and CXR scans

\begin{tabular}{llllll}
\hline \multirow{2}{*}{$\begin{array}{l}\text { Evaluation } \\
\text { Matrices }\end{array}$} & \multicolumn{2}{c}{ CT } & & \multicolumn{2}{c}{ CXR } \\
\cline { 2 - 3 } $\begin{array}{l}\text { COVID-19, non- } \\
\text { COVID-19 and } \\
\text { pneumonia }\end{array}$ & $\begin{array}{l}\text { COVID-19 and } \\
\text { non-COVID-19 }\end{array}$ & $\begin{array}{l}\text { COVID-19, non- } \\
\text { COVID-19 and } \\
\text { pneumonia }\end{array}$ & $\begin{array}{l}\text { COVID-19 and } \\
\text { non-COVID-19 }\end{array}$ \\
\hline Accuracy & $100 \%$ & $98.85 \%$ & $96.85 \%$ & $100 \%$ \\
Specificity & $100 \%$ & $100 \%$ & $97.40 \%$ & $100 \%$ \\
Sensitivity & $100 \%$ & $99.33 \%$ & $96.30 \%$ & $100 \%$ \\
Precision & $100 \%$ & $99 \%$ & $97.37 \%$ & $100 \%$ \\
NPV & $100 \%$ & $99.32 \%$ & $96.34 \%$ & $100 \%$ \\
F1 score & $100 \%$ & $99.62 \%$ & $96.83 \%$ & $100 \%$ \\
MCC & $100 \%$ & $99.33 \%$ & $93.71 \%$ & $100 \%$ \\
\hline
\end{tabular}


The classification performance of the proposed CNN-based COVID-19 detection model in terms of various measures like accuracy, specificity, sensitivity, and precision, NPV, F1-score, and MCC using CXR scans are summarized in Tab. 3. The model outcomes demonstrated the classification performance of COVID-19, non-COVID-19 and pneumonia using CXR scans as 96.85\% overall accuracy, 97.40\% specificity, 96.30\% sensitivity, 97.37\% precision, 96.34\% NPV, 96.83\% for F1-score, and 93.71\% for MCC. On the other side, COVID-19 and non-COVID-19 detection classification using CXR scans demonstrated superior performance represented as 100\% overall accuracy, $100 \%$ specificity, $100 \%$ sensitivity, $100 \%$ precision, $100 \%$ NPV, 1 for F1-score, and 1 for MCC. Therefore, the proposed network gives high results with few CXR input scans. The confusion matrix is used to clarify the prediction ratio for different classification, as shown in Fig. 6. The confusion matrix is based on the false positive rate and true negative rate considering balanced distribution in the dataset, which improved the prediction ratio and enhanced the model performance. It was found that the proposed model gives better and consistent true positive and true negative scores, which can efficiently classify COVID-19 cases.

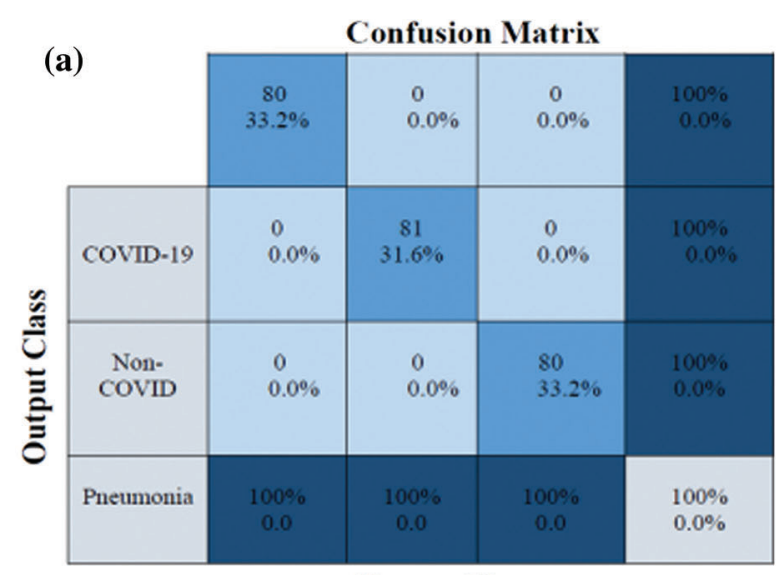

Target Class

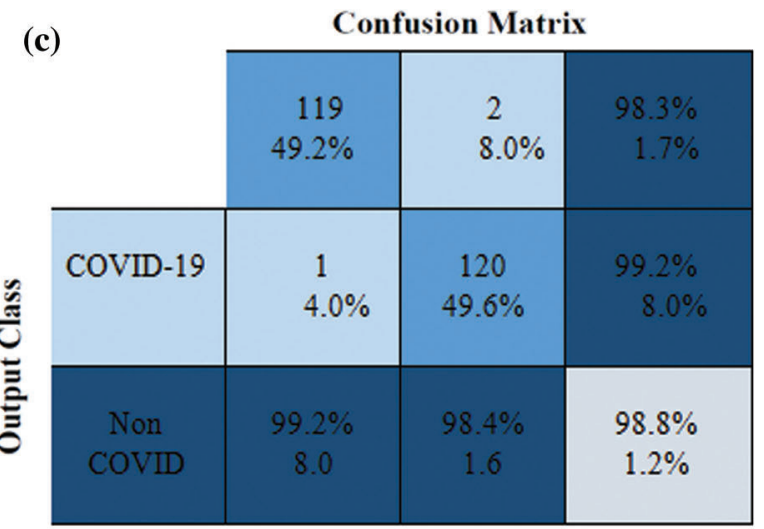

Target Class

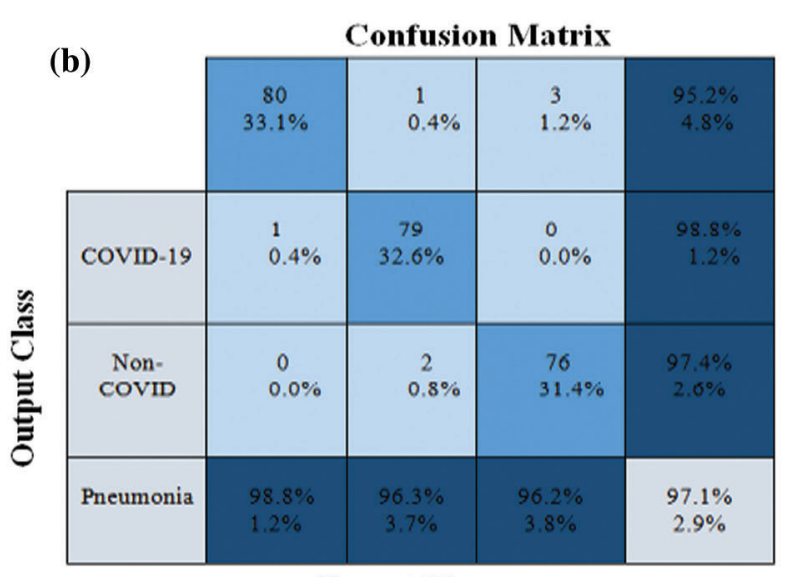

Target Class

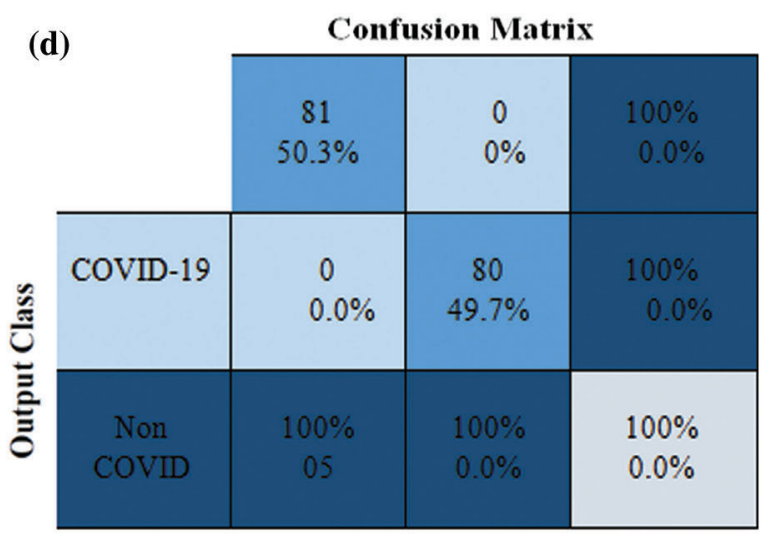

Target Class

Figure 6: Confusion matrix of the classification process (a) COVID-19, non-COVID-19, and pneumonia using CT scans, (b) COVID-19, non-COVID-19, and pneumonia using CXR scans, (c) COVID-19 and non-COVID-19 and pneumonia using CT scans (d) COVID-19 and non-COVID-19 using CXR scans

Moreover, the proposed CNN-based COVID-19 detection model performance is evaluated in terms of accuracy and cross-entropy (loss) for both the training and testing stages. Fig. 7 shows the model accuracy 
and the loss of classification for COVID-19, non-COVID-19, and pneumonia using CT scans. The training and testing accuracies are $100 \%$ and $100 \%$, respectively, at iterations number equals 1600 . The training and testing loss are 0.019 and 0.16 , respectively, at iterations number equals to 1600 . Fig. 8 shows the model accuracy and the losses of classification for COVID-19 and non-COVID-19 using CT scans. The training and testing accuracies are $100 \%$ and $98.85 \%$, respectively, at iterations number equals 1600 . The training and testing losses are 0.02 and 0.29 , respectively, at iterations number equals to 1600. Fig. 9 shows the model accuracy and the losses of classification for COVID-19, non-COVID-19, and pneumonia using CXR scans. The training and testing accuracies are $100 \%$ and $96.85 \%$, respectively, at iterations number equals 1600 . The training and testing losses are 0.01 and 0.28 , respectively, at iterations number equals to 1600. Fig. 10 shows the model accuracy and the loss of classification for COVID-19 and non-COVID-19 using CXR scans. The training and testing accuracies are $100 \%$ and $100 \%$, respectively, at iterations number equals 1600 .

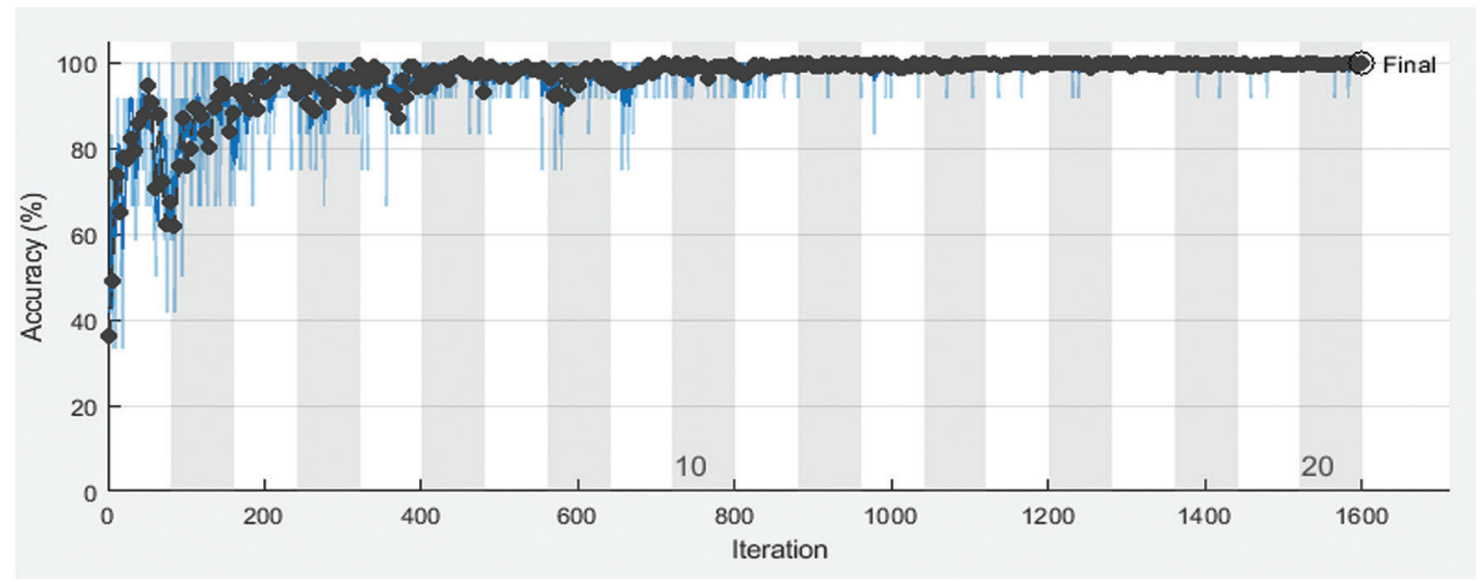

(a)

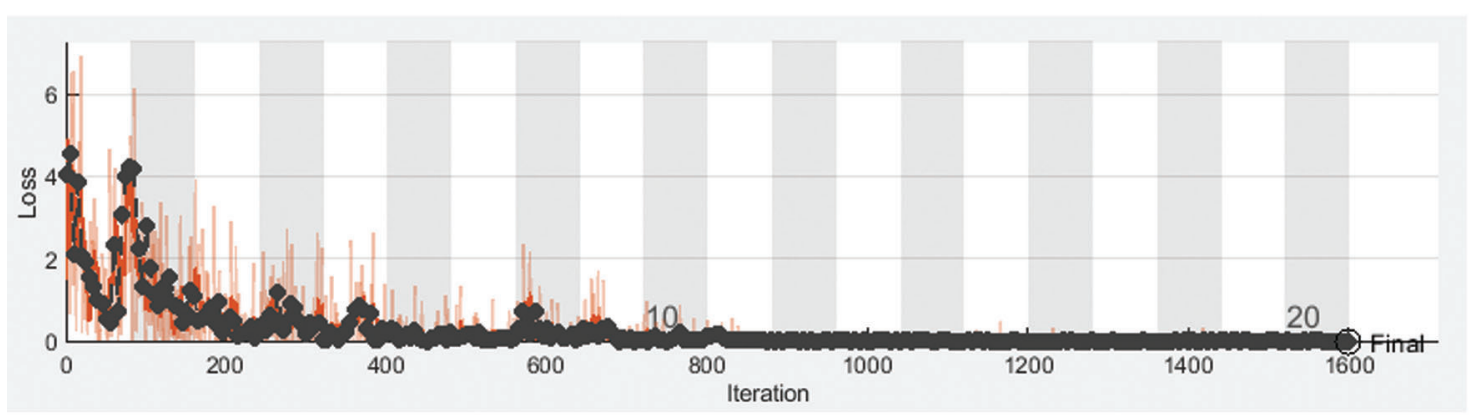

(b)

Figure 7: The model accuracy and loss for COVID-19, non-COVID-19, and pneumonia using CT scans (a) The accuracy Model (b) The loss Model

Similarly, the training and testing losses are 0.01 and 0.13 , respectively, at iterations number equals 1600. The proposed model has significant effects on detecting COVID-19 based on either CT or CXR scans by analyzing the obtained results. The suggested network can conduct better feature extraction using CT scans and detect COVID-19 from non-COVID-19 (normal) and pneumonia cases with maximum accuracy. Also, the suggested network can conduct better feature extraction using CXR scans and can detect COVID-19 from non-COVID-19 cases with maximum accuracy. Therefore, the proposed 
work recommended for high accuracy of COVID-19 detection using an extensive dataset of CT scans or less dataset of CXR scans.

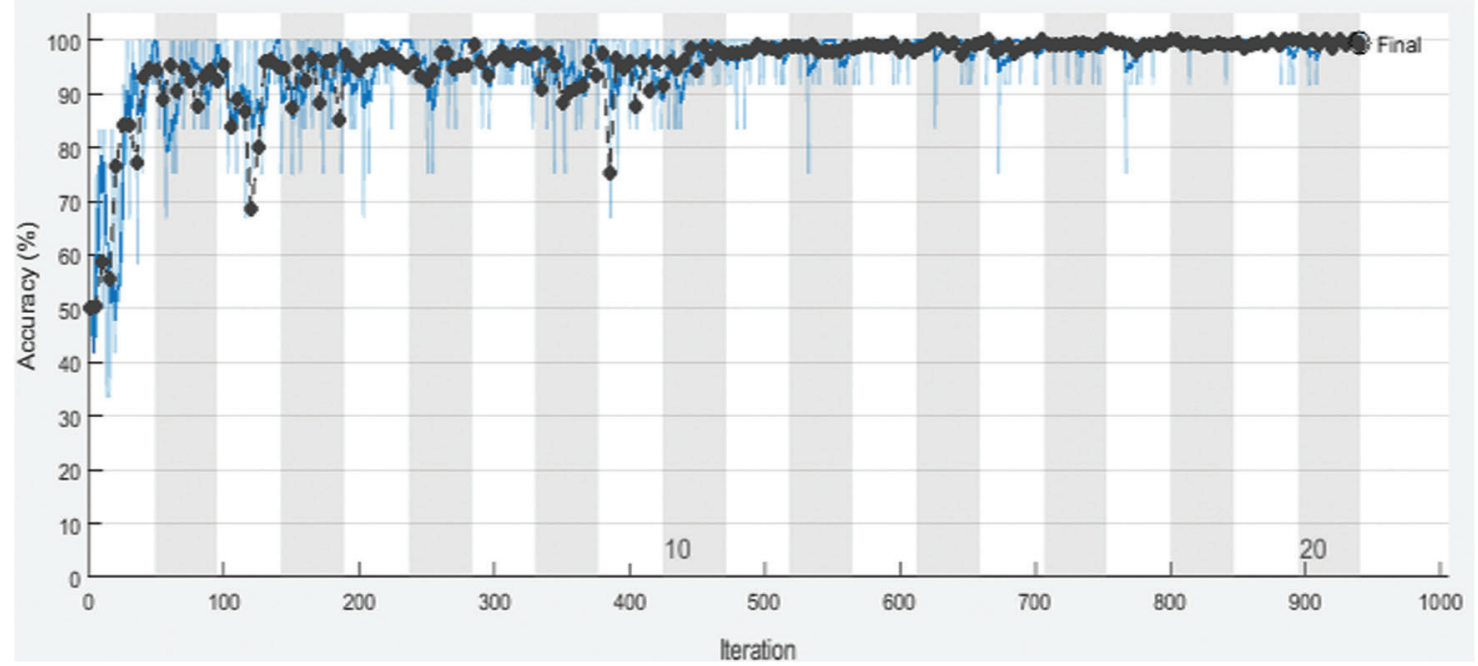

(a)

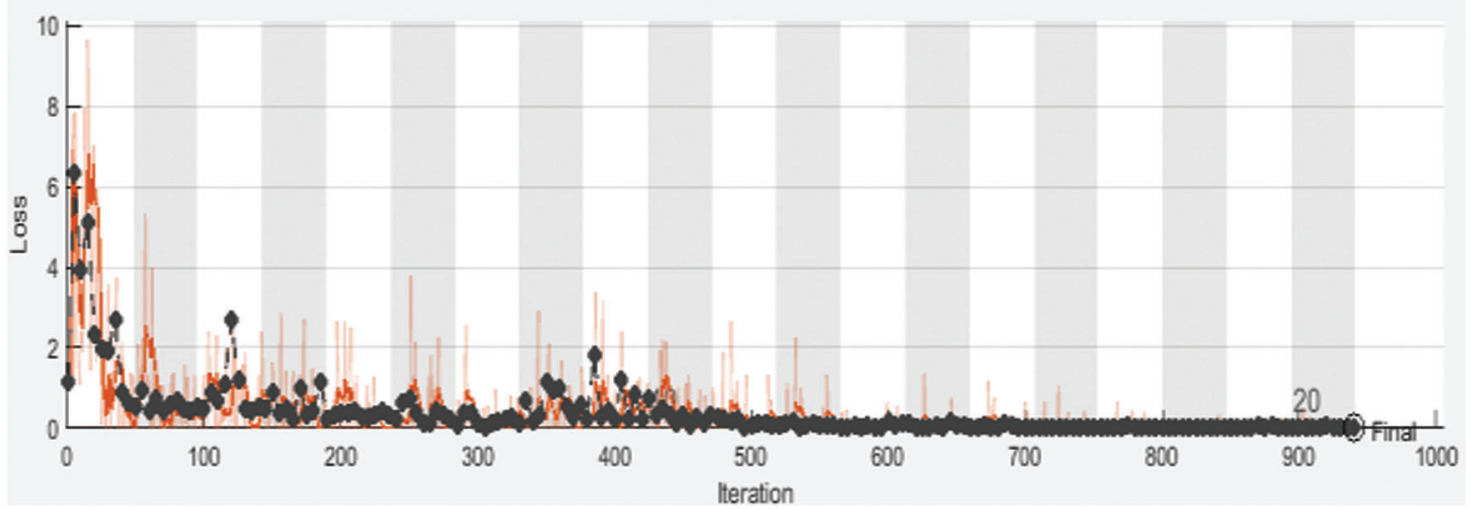

(b)

Figure 8: The model accuracy and loss for COVID-19 and non-COVID-19 using CT scans (a) The accuracy Model (b) The loss Model 


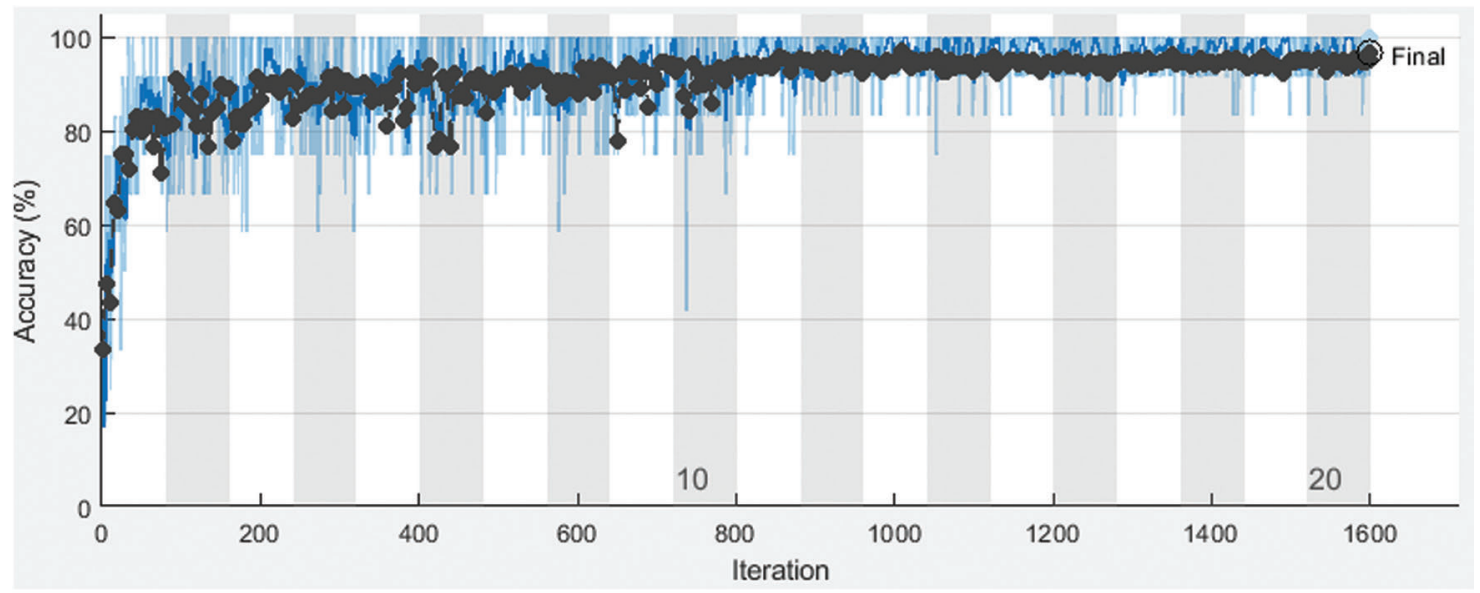

(a)

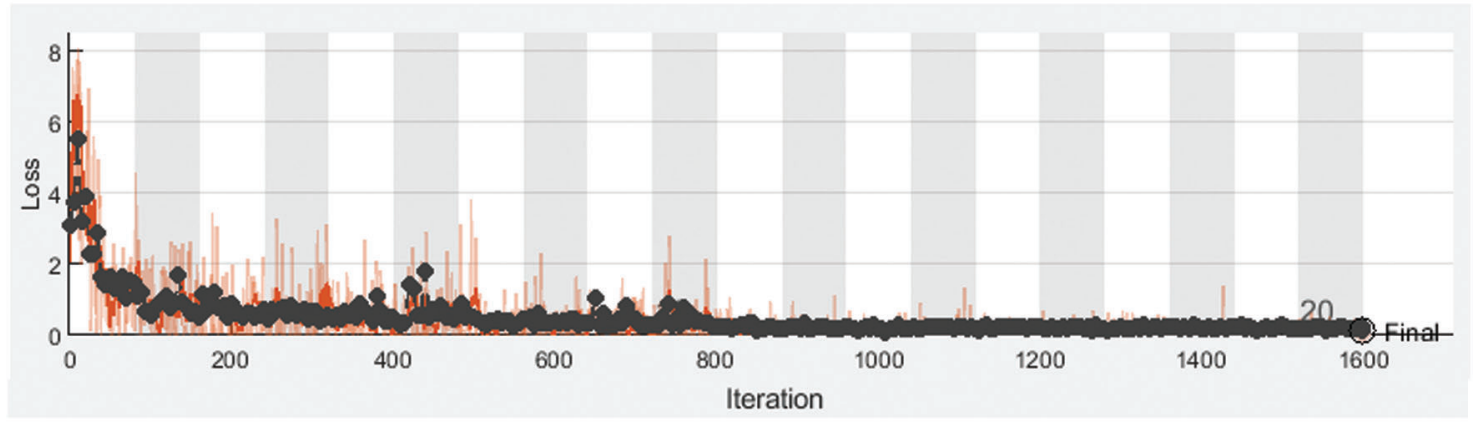

(b)

Figure 9: The model accuracy and loss for COVID-19, non-COVID-19, and pneumonia using CXR scans (a) The accuracy Model (b) The loss Model 


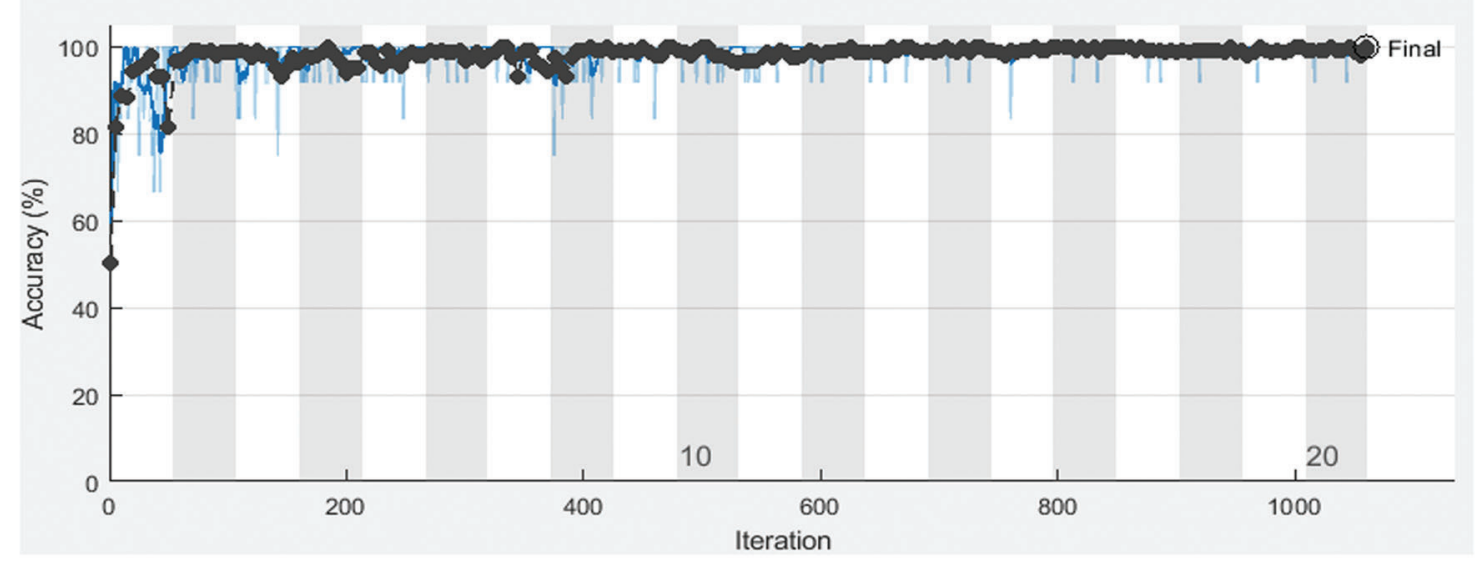

(a)

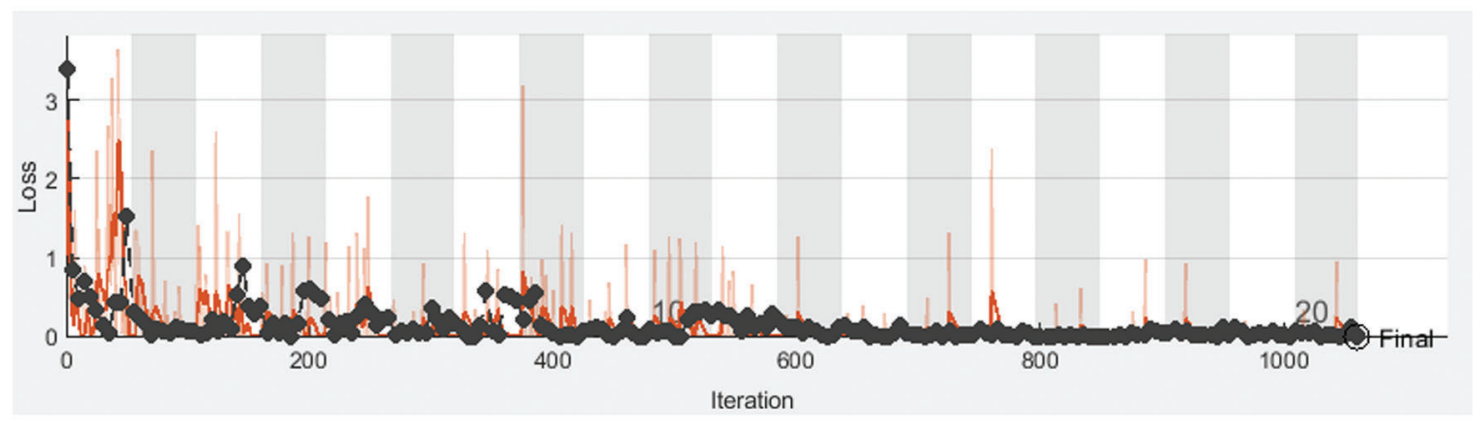

(b)

Figure 10: The model accuracy and loss for COVID-19 and non-COVID-19 using CXR scans (a) The accuracy Model (b) The loss Model

\section{Conclusions}

During the COVID-19 health emergency, it is imperative to identify the positive cases rapidly. This work proposed a CNN-based COVID-19 detection model using CT and CXR scans. The proposed model consists of two stages, feature extraction used to capture the detailed characteristics, and classification which utilizes the knowledge built in the previous stage to decide for COVID-19 detection. The performance of the proposed CNN model for the COVID-19 detection model is evaluated using different statistical metrics like specificity, sensitivity, precision, MCC, F1 score, and NPV. The outcomes of this evaluation demonstrated incomparable results, which highlight that, the proposed model can efficiently handle different types of images and achieve high accuracy for the binary and multi-classification processes using both CT and CXR scans.

Acknowledgement: The authors would like to thank the Deanship of Scientific Research, Taif University, Saudi Arabia, for funding research project number 1-441-61.

Funding Statement: This research was supported by the Deanship of Scientific Research, Taif University, Saudi Arabia, under research project number 1-441-61.

Conflicts of Interest: The authors declare that they have no conflicts of interest to report regarding the present study. 


\section{References}

[1] World Health Organization, Pneumonia of unknown cause-China: Emergencies preparedness, response. Geneva: World Health Organization, 2020.

[2] World Health Organization, Coronavirus disease 2019 (COVID-19) situation report-76. Geneva: World Health Organization, 2020.

[3] Z. Y. Zu, M. D. Jiang, P. P. Xu, W. Chen, Q. Q. Ni et al., "Coronavirus disease 2019 (COVID-19): A perspective from China," Radiology, vol. 296, no. 2, pp. E15-E25, 2020.

[4] Q. Hu, H. Guan, Z. Sun, L. Huang, C. Chen et al., "Early CT features and temporal lung changes in COVID-19 pneumonia in Wuhan," China European Journal of Radiology, vol. 128, pp. 109017, 2020.

[5] F. Wu, S. Zhao, B. Yu, Y. Chen, W. Wang et al., "A new coronavirus associated with human respiratory disease in China," Nature, vol. 579, pp. 265-269, 2020.

[6] T. Liang, "Handbook of COVID-19 prevention and treatment," Zhejiang University, 2020. [Online]. Available: http://www.zju.edu.cn/english/2020/0323/c19573a1987520/page.htm.

[7] M. Cascella, M. Rajnik, A. Cuomo, S. C. Dulebohn and R. D. Napoli, Features, evaluation and treatment coronavirus (COVID-19). StatPearls Publishing, 2020. [Online]. Available: https:/www.ncbi.nlm.nih.gov/ books/NBK554776/.

[8] A. Tahamtan and A. Ardebili, "Real-time RT-PCR in COVID-19 detection: Issues affecting the results," Expert Review of Molecular Diagnostics, vol. 20, no. 5, pp. 453-454, 2020.

[9] J. Lascarrou, G. Colin, A. L. Thuaut, N. Serck, M. Ohana et al., "Predictors of negative first SARS-CoV-2 RT-PCR despite final diagnosis of COVID-19 and association with outcome," Scientific Reports, vol. 11, pp. 2388, 2021.

[10] S. Abbas, A. Rafique, B. Abbas, R. Iqbal and P. J. Med, "Real-time polymerase chain reaction trends in COVID-19 patients," Pakistan Journal of Medical Sciences, vol. 37, no. 1, pp. 180-184, 2021.

[11] A. Jacobi, M. Chung, A. Bernheim and C. Eber, "Portable chest X-ray in coronavirus disease-19 (COVID-19): A pictorial review," Clinical Imaging, vol. 64, pp. 35-42, 2020.

[12] H. Y. F. Wong, H. Y. S. Lam, A. H. T. Fong, S. T. Leung, T. W. Y. Chin et al., "Frequency and distribution of chest radiographic findings in COVID-19 positive patients," Radiology, vol. 296, no. 2, pp. E72-E78, 2020.

[13] C. Czawlytko, R. Hossain and C. S. White, "COVID-19 diagnostic imaging recommendations," Applied Radiology, vol. 49, no. 3, pp. 10-15, 2020.

[14] J. Fu, J. Wang, W. Guo and P. Peng, "Multi-mounted X-ray cone-beam computed tomography," Nuclear Instruments and Methods in Physics Research, vol. 888, pp. 119-125, 2018.

[15] S. Cho, S. Lim, C. Kim, S. Wi, T. Kwon et al., "Enhancement of soft-tissue contrast in cone-beam CT using an anti-scatter grid with a sparse sampling approach," Medical Physics, vol. 70, pp. 1-9, 2020.

[16] A. Ibrahim, S. Mohammed and H. A. Ali, "Breast cancer detection and classification using thermography: A review," in Int. Conf. on Advanced Machine Learning Technologies and Applications, AMLTA 2018. Advances in Intelligent Systems and Computing, Springer, Cham, vol. 723, pp. 496-505, 2018.

[17] P. Lei, Z. Huang, G. Liu, P. Wang, W. Song et al., "Clinical and computed tomographic (CT) images characteristics in the patients with COVID-19 infection: What should radiologists need to know," Journal of X-Ray Science and Technology, vol. 28, no. 3, pp. 369-381, 2020.

[18] A. Bernheim, X. Mei, M. Huang, Y. Yang, Z. A. Fayad et al., "Chest CT findings in coronavirus disease-19 (COVID-19): Relationship to duration of infection," Radiology, vol. 295, no. 3, pp. 685-691, 2020.

[19] K. Li, J. Wu, F. Wu, D. Guo, L. Chen et al., "The clinical and chest CT features associated with severe and critical COVID-19 pneumonia," Investigative Radiology, vol. 55, no. 6, pp. 327-331, 2020.

[20] K. Li, Y. Fang, W. Li, C. Pan, P. Qin et al., "CT image visual quantitative evaluation and clinical classification of coronavirus disease (COVID-19)," European Radiology, vol. 30, pp. 4407-4416, 2020.

[21] P. M. Shakeel, M. Burhanuddin and M. I. Desa, "Automatic lung cancer detection from CT image using improved deep neural network and ensemble classifier," Neural Computing and Applications, pp. 1-14, 2020.

[22] Y. LeCun, Y. Bengio and G. Hinton, “Deep learning," Nature, vol. 521, pp. 436-444, 2015.

[23] A. Esteva, B. Kuprel, R. A. Novoa, J. Ko and S. M. Swetter, "Dermatologist-level classification of skin cancer with deep neural networks," Nature, vol. 542, pp. 115-118, 2017. 
[24] M. Abd Elaziz, K. M. Hosny, A. Salah, M. M. Darwish, S. Lu et al., "New machine learning method for image based diagnosis of COVID-19," PLoS One, vol. 15, no. 6, pp. e0235187, 2020.

[25] S. Wang, B. Kang, J. Ma, X. Zeng, M. Xiao et al., "A deep learning algorithm using CT images to screen for coronavirus disease (COVID-19)," European Radiology, pp. 1-9, 2021.

[26] L. Wang, Z. Q. Lin and A. Wong, "COVID-net: A tailored deep convolutional neural network design for detection of COVID-19 cases from chest X-ray images," Scientific Reports, vol. 10, pp. 19549, 2020.

[27] P. Kumar and S. Kumari, "Detection of coronavirus disease (COVID-19) based on deep features," Preprints, 2020030300, pp. 1-10, 2020.

[28] I. U. Khan and N. Aslam, "A deep-learning-based framework for automated diagnosis of COVID-19 using X-ray images," Information, vol. 11, no. 9, pp. 419, 2020.

[29] A. Jaiswal and A. S. Bist, "Analysis of deep learning algorithms on COVID-19 radiography database," International Journal of Advanced Science and Technology, vol. 29, no. 11, pp. 1268-1275, 2020.

[30] M. Nour, Z. Cömert and K. Polat, "A novel medical diagnosis model for COVID-19 infection detection based on deep features and Bayesian optimization," Applied Soft Computing, vol. 97, pp. 106580, 2020.

[31] T. Ozturk, M. Talo, E. A. Yildirim, U. B. Baloglu, O. Yildirim et al., "Automated detection of COVID-19 cases using deep neural networks with X-ray images," Computers in Biology and Medicine, vol. 121, pp. 103792, 2020.

[32] A. I. Khan, J. L. Shah and M. M. Bhat, "Coronet: A deep neural network for detection and diagnosis of COVID-19 from chest X-ray images," Computer Methods and Programs in Biomedicine, vol. 196, pp. 105581, 2020.

[33] I. D. Apostolopoulos and T. A. Mpesiana, "COVID-19: Automatic detection from X-ray images utilizing transfer learning with convolutional neural networks," Physical and Engineering Sciences in Medicine, vol. 43, pp. 635-640, 2020.

[34] X. Xu, X. Jiang, C. Ma, P. Du, X. Li et al., "A deep learning system to screen novel coronavirus disease 2019 pneumonia," Engineering, vol. 6, no. 10, pp. 1122-1129, 2020.

[35] L. Li, L. Qin, Z. Xu, Y. Yin, X. Wang et al., "Using artificial intelligence to detect COVID-19 and communityacquired pneumonia based on pulmonary CT: Evaluation of the diagnostic accuracy," Radiology, vol. 296, no. 2, pp. E65-E71, 2020.

[36] S. Ying, S. Zheng, L. Li, X. Zhang, X. Zhang et al., "Deep learning enables accurate diagnosis of novel coronavirus (COVID-19) with CT images," medRxiv, IEEE/ACM Transactions On Computational Biology and Bioinformatics, 2020.

[37] B. Ghoshal and A. Tucker, "Estimating uncertainty and interpretability in deep learning for coronavirus (COVID-19) detection," arXiv: 2003.10769v2, preprint, 2020.

[38] C. Ouchichaa, O. Ammor and M. Meknassi, "CVDNet: A novel deep learning architecture for detection of coronavirus (COVID-19) from chest X-ray images," Chaos, Solitons \& Fractals, vol. 140, pp. 110245, 2020.

[39] M. Heidari, S. Mirniaharikandehei, A. Z. Khuzani, G. Danala, Y. Qiu et al., "Improving the performance of CNN to predict the likelihood of COVID-19 using chest X-ray images with preprocessing algorithms," International Journal of Medical Informatics, vol. 144, pp. 104284, 2020.

[40] M. Polsinellia, L. Cinqueb and G. Placidia, "A light CNN for detecting COVID-19 from CT scans of the chest," Pattern Recognition Letters, vol. 140, pp. 95-100, 2020.

[41] L. L. Wang, K. Lo, Y. Chandrasekhar, R. Reas, J. Yang et al., "CORD-19: The COVID-19 open research dataset," arXiv: 2004.10706v2, preprint, 2020.

[42] J. Zhang, Y. Xie, Y. Li, C. Shen and Y. Xia, "COVID-19 screening on chest X-ray images using deep learning based anomaly detection," arXiv: 2003.12338, preprint, 2020.

[43] K. He, X. Zhang, S. Ren and J. Sun, "Deep residual learning for image recognition," in IEEE Conf. on Computer Vision and Pattern Recognition (CVPR), USA, pp. 770-778, 2016.

[44] N. Tajbakhsh, J. Y. Shin, S. R. Gurudu, R. T. Hurst, C. B. Kendall et al., "Convolutional neural networks for medical image analysis: Full training or fine tuning," IEEE Transactions on Medical Imaging, vol. 35, pp. 1299-1312, 2016.

[45] A. M. Hasan, H. A. Jalab, F. Meziane, H. Kahtan and A. S. Al-Ahmad, "Combining deep and handcrafted image features for MRI brain scan classification,” IEEE Access, vol. 7, pp. 79959-79967, 2019. 